\title{
Knowledge Medium for the Global Design
}

\author{
Toshiharu Taura ${ }^{1}$, Yasukazu Aoki ${ }^{2}$, Masanobu Muranaka ${ }^{3}$, Terueki Ogawa ${ }^{4}$, \\ Yoshinori Kohno ${ }^{5}$, Tominori Yamada ${ }^{6}$, Yutaka Yamada ${ }^{7}$ \\ ${ }^{1}$ RACE, the University of Tokyo, 4-6-1, Komaba, Meguro-ku, Tokyo 153-8904, Japan \\ ${ }^{2}$ Duo Systems Co., Ltd., Waseda-Tsurumakicho 518, Shinjuku-ku, Tokyo 162-0041, Japan \\ ${ }^{3}$ Nippon Steel Corp., 2-31-1, Shinkawa, Chuo-ku, Tokyo 104-0033, Japan \\ ${ }^{4}$ Sony Systems Design Corp. (Present affiliation: Sony Chemicals Corp., 1-6-3, Nihonbashi-Muromachi, \\ Chuo-ku, Tokyo 103-8312, Japan) \\ ${ }^{5}$ Mitsui Engineering \& Shipbuilding Co., Ltd., 1-3-D7, Nakase, Mihama-ku, Chiba-city, Chiba 261-0023, Japan \\ ${ }^{6}$ Mitsui Engineering \& Shipbuilding Co., Ltd. (Present affiliation: Tubal-Cain Engineering consultant, \\ Kou 3882, Komoro-city, Nagano 384-0023, Japan) \\ ${ }^{7}$ Nippon Unisoft Corp., 2-13-9, Nihonbashi-Ningyocho, Chuo-ku, Tokyo 103-0013, Japan
}

\begin{abstract}
This paper describes a new concept "Global Design," and proposes a system realizing this concept. The system is based on the "Activity Chain Model" which can easily capture, accumulate and transfer dynamic design information including histories of products and intent by focusing on design activities and links between them.
\end{abstract}

\section{INTRODUCTION}

\section{A. Globalization of design}

Today's computer network technology enables industrial activities without the restrictions of physical distance or time, and many enterprises have adopted a global production style based on international cooperation. However, when design and manufacturing in a production process are considered separately, the design process is still performed locally by direct face-to-face communication even though globalization of the manufacturing process has become common. It is an important issue in future production industries to attain globalization of design.

The authors consider the globalization of design as follows. One is "spatial globalization" of design. This includes cooperation among designers with various abilities to design various product families. This also includes international division of the design process into overseas markets or factories to realize short-term development of products. Another is "time globalization" of design. This includes the reference and the reuse of past designs as examples. This also includes accumulation of design expertise for succession of designers' skills. In this paper, the new design style suitable for the globalization of design as stated above is called "global design."

\section{B. Necessity for new design-support technologies}

From the viewpoint of an information system, most design information treated by the system is not for design but for manufacturing. There are no established criteria on what information should be managed and provided in what way to support creative and effective design.

Advancement of design-support tools, such as 3dimensional $\mathrm{CAD}$, makes it easier than ever to describe geometric information or attribute information based on a result of a design. However, it is difficult to express the designer's own ideas and recognition of problems using these design-support tools. There is still no tool that can support the designer's work itself. Moreover, industrial standards, such as CALS and STEP[1], are the minimum necessary framework for sharing product information among the users of such information, and are not aimed at information sharing for the sake of creativity and efficiency in the design process.

It can be said that the realization of an informationsharing framework to make design work global is one of the most important future research subjects.

\section{OBJECTIVES}

In this paper, the computer medium for supporting global design is called the "knowledge medium." The knowledge medium for supporting global design must provide an information-sharing mechanism that exceeds the ability of conventional media in the range and methods of handling information.

First, in the range of information that the knowledge medium handles, not only product information such as drawings, but also the histories and the intent of the design, which have been difficult for conventional media to handle, should be contained. Next, as a method by which the knowledge medium handles information, the knowledge medium must perform simultaneous capture, accumulation and transfer of the design information through one medium; the conventional media perform those processes separately. Furthermore, it is also important for the knowledge medium to mitigate the designer's overhead of inputting information by capturing support concerning the history and the intent of the design.

Thus, the knowledge medium which supports global design is a computer medium for designers to share dynamically the wide range of design information containing the history and the intent, and is a new kind of designsupport environment in which designers can interact with each other through a shared design process. 
The objectives of this paper are to propose the concept of the knowledge medium, and to introduce the design information model necessary for realizing the knowledge medium in a computer system.

\section{DESIGN INFORMATION MODEL}

\section{A. Background}

Now, toward the realization of the cooperative designsupport environment through information sharing, utilization of the information technologies, including the PDM system, has become more common than ever before in design work[13]. However, in PDM, management of design output (drawings, CAD information, design documents, etc.) is considered important, and the information on the history and the intent of the design are restricted to the range necessary for the management of the design output.

The authors start the discussion by paying attention to the manner of intent expression. Garcia et al. [8] classified the cooperative design-support environment into three methods based on the manner of intent expression:

(1) the knowledge-model-based method,

(2) the argument-based method,

(3) the action-based method.

They compared these three methods from the viewpoint of intent-capturing capability and intent-explanation capability. Their comparison showed that the explanation of the design intent is better in the knowledge-model-based method, the argument-based method, and the action-based method, in that order. In addition, it is shown that capturing the design intent is easier in the action-based method, the argument-based method, and the knowledge-model-based method, in that order.

The description model of the design intent in SHARE[17], SHADE[4][5] and n-dim[6] assumes the use of the conventional method that includes the knowledge-modelbased method or the argument-based method. In the description model of the design intent using the knowledgemodel-based method, although the intent-explanation capability is better, capturing the design intent is difficult. In particular, in a creative field in which the designers themselves cannot denote the knowledge or the rule on the design, there is the problem that it becomes harder to capture the design intent in a knowledge model.

It is possible for gIBIS[7][8][9] and PHI[9][11][12], which are based on the description model of design intent using the argument-based method, to describe the design intent, even in a field in which it is difficult to describe a knowledge model, and to give an explanation based on the intent. However, in the argument-based method, capturing the design intent is not necessarily easy since designers must register proposals, arguments, opinions, and the relations among them into the system during design work. ADD[8] and DRIM[15] study active capturing support of the design intent by the computer using the argument-based method. However, it is difficult for the system to support capturing of the design intent unless the system can understand the designer's knowledge or rule concerning the domain of the design. Moreover, from the viewpoint of the explanation capability of the intent according to the classification of the process explanation by Wright[17], although the intentdescription model using the argument-based method is suitable for "teleological explanation," which is an explanation of the reason for an action according to the future state to be satisfied, it is not necessarily suitable for "causal explanation," which is an explanation of the reason for an action according to the past state.

In the preceding study by some of the authors[16], a new intent-description model using the action-based method, which extends the explanation capability of the intentdescription model using the argument-based method to "causal explanation," was proposed. Generally, in the intentdescription model using an action-based method, such as Electronic Notebook[9], since the action itself serves as the explanation of the intent, although capturing the design intent is easy, the intent-explanation capability is not satisfactory. This paper shows a design information model called the "Activity Chain Model" that introduces the chained structure into the action in order to improve the explanation capability based on the intent, and which employs easy and efficient capturing of the design intent in the action-based method.

\section{B. Definition and hypothesis of the Activity Chain Model}

In the following, the description method of the history and the intent of the design based on the Activity Chain Model are explained in detail. We define the following terms.

(1) "Product Information" means the information on the result of the design (an intermediate result is also included) such as drawings, CAD information, or design documents.

(2) "Design Process Information" consists of the information on the history and the intent of the design.

(3) "Product Unit" means the unit of product information that corresponds to a part or an assembly.

(4) "Design Activity" means the external action that can be described as operations on the product information. Inner (mental) action of the designer is not included.

(5) "Activity Unit" means the unit of design activity that corresponds to each decision on the specifications or the shape of the product by the designer.

\section{1) Description of the history of the design process}

In the Activity Chain Model, the information on the history of the design processes includes product information as an attribute, and the design process information and the product information are integrated with the focus on the design process information.

First, for the product information, we consider a "composition relation" including "child-parts and a parentpart" relation. The product information is described by the product units that are associated with each other through the composition relations. Next, for the design activity, we consider a "sequence relation" according to the order of execution of design activities along the time axis. The information about the history of the design is described by the activity units associated with each other through the 


\section{Time-1}

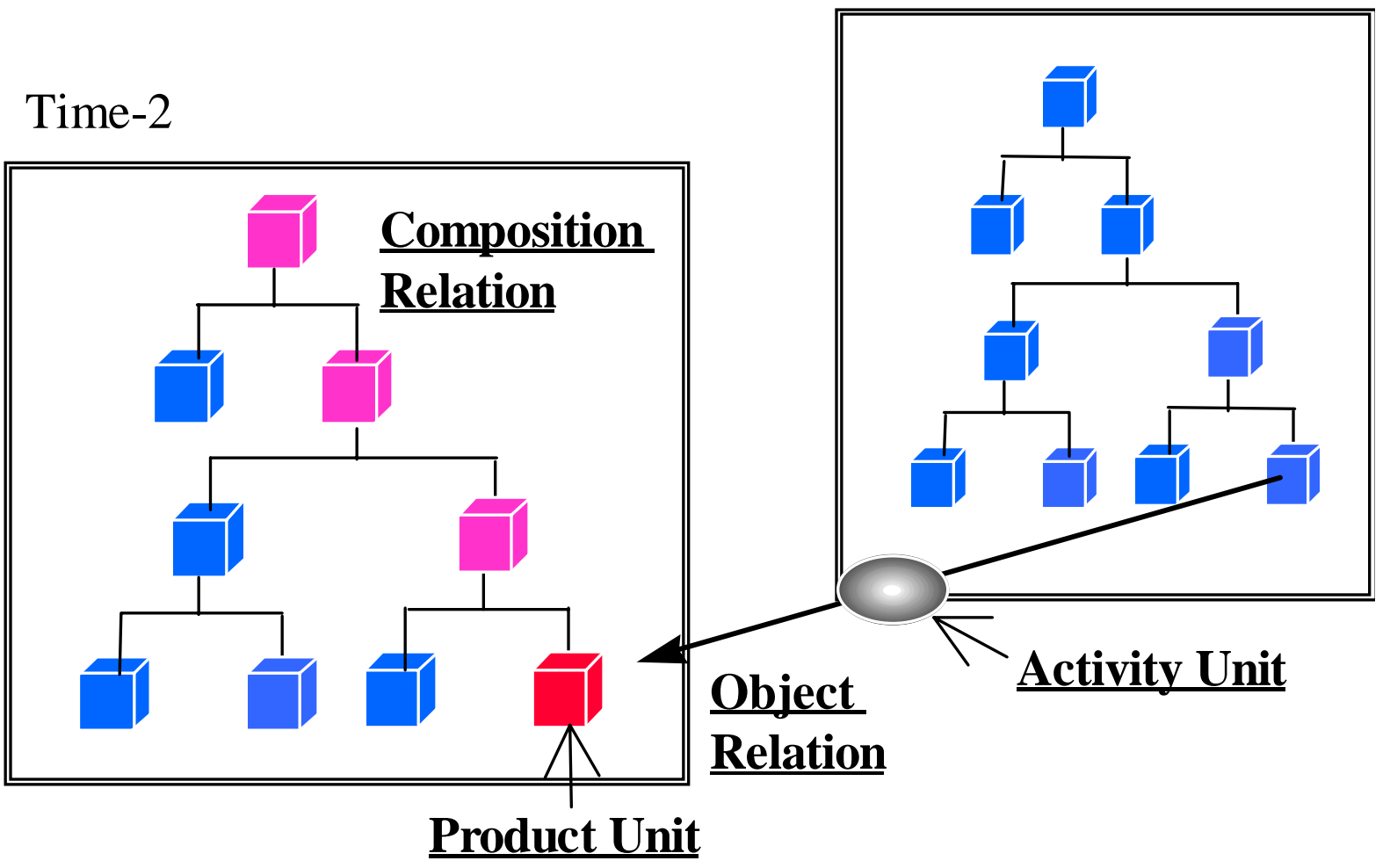

Fig. 1. Product Units and Activity Units.

sequence relations. Between a product unit and an activity unit, we consider the "object relation" which means that the object of the design process denoted by the activity unit is the product unit.

In this way, the Activity Chain Model describes the history of the design by means of the product units and their composition relations, the activity units and their sequence relations, and the object relations between them (Fig. 1).

\section{2) Description of the design intent}

In the Activity Chain Model, we also consider a "constraint relation" and an "alternative relation" between the activity units as the relations concerning the design intent (Fig. 2). Moreover, a "reason" is described as an attribute of these relations. The reason here denotes not the reason for the activity but the viewpoint of the designer in determining the constraint relation or the alternative relation. The reason for the activity is expressed by the entire structure of the constraint relation and the alternative relation, which are related to the activity unit, including reason (viewpoint) as their attribute.

The constraint relation denotes that a certain activity unit in the past is a constraint of a succeeding activity unit, and it is described as a multiple-to-multiple relation. Using the "causal reason," which is an attribute of the constraint relation, the designers can describe the reason why they consider the activity unit to be a constraint. The alternative relation denotes that a certain activity unit is an alternative of another activity unit, and it is described as a multiple-tomultiple relation. Using the "teleological reason," which is an attribute of the alternative relation, the designers can describe the reason why they actually adopt (or do not adopt) the activity unit in comparison with its alternative.

Thus, the Activity Chain Model describes the design intent by means of association between the activity units using the constraint relations, their causal reasons, the alternative relations and their teleological reasons. The hypothesis underlying the Activity Chain Model is that the designer's inner (mental) process which forms the design intent can be represented as a chain structure of the external activity units. The class diagram of the Activity Chain Model

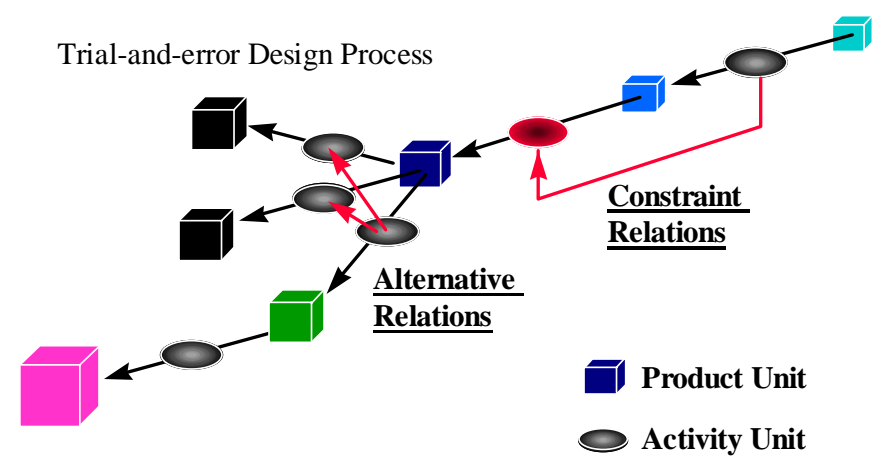

Fig. 2. Constraint Relations and Alternative Relations. 


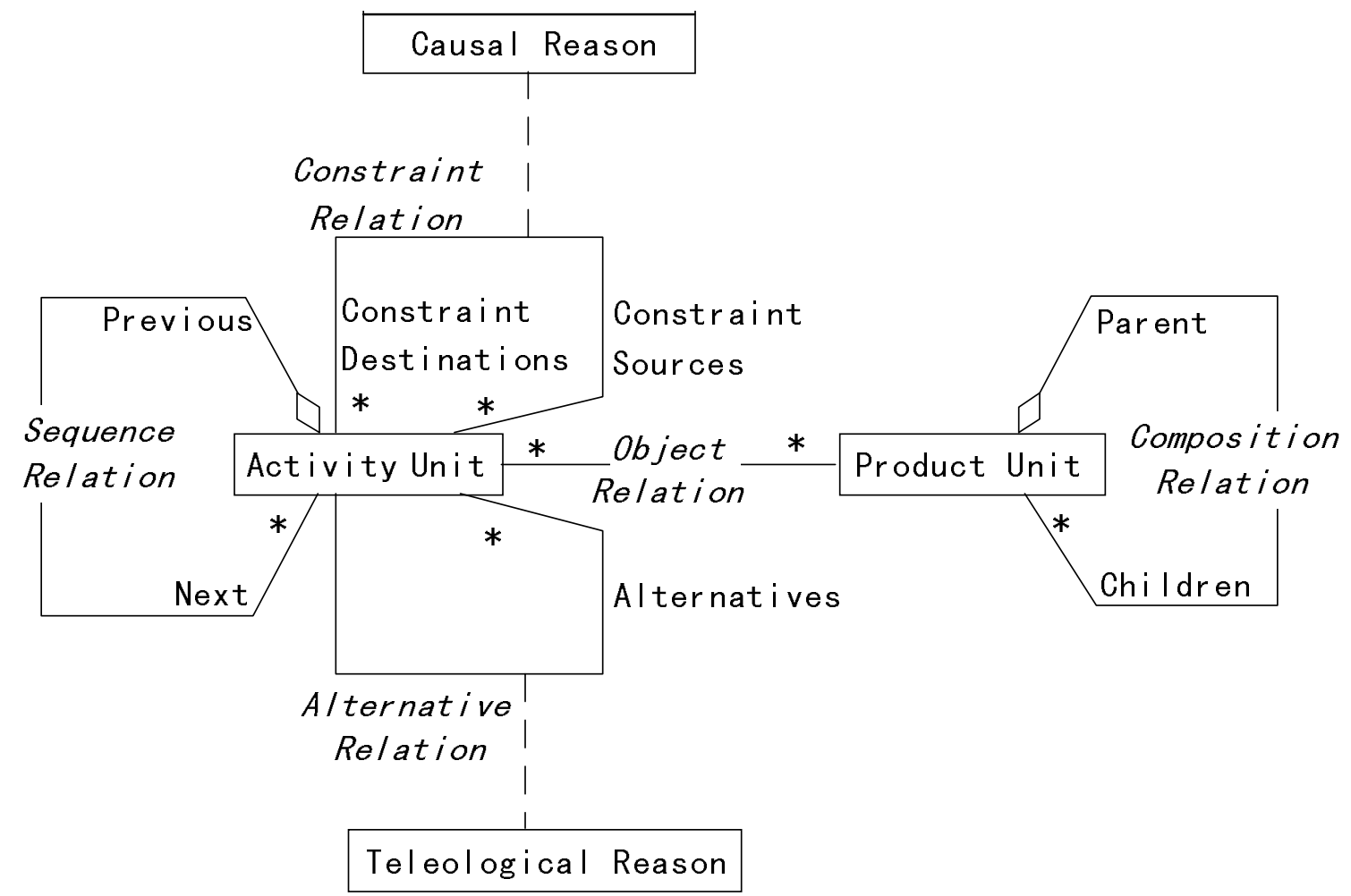

Fig. 3. A Class Diagram of the Activity Chain Model.

is shown in Fig. 3 according to the UML (unified modeling language) notation[7].

\section{Method of handling the design process information}

\section{1) Support for capturing design process information}

Since it is important for the argument-based intentdescription model to capture the design intent efficiently, ADD [8] and DRIM[15] provide active support for capturing the design intent using the dependence among the decisions, the knowledge and the rules on the domain of the design. On the other hand, active capturing support of design process information, including the design intent, by the Activity Chain Model focuses on the derivation of relations between design activities and attributes of the relations. The details are described in the following.

First, in order to support the capture of the design history, it is sufficient to generate, without extra input from the designer, the activity units corresponding to the designer's operations, the sequence relation between the activity units along the time axis, and the object relation between the activity unit and the product unit. Next, when a designer inputs the constraint relation and the alternative relation explicitly, it is effective to choose the constraint relation from the activity units in the past designs, and the alternative relation from the activity units in the past trial-and-error histories. In the Activity Chain Model, the structure of the chains between the activity units described by the constraint relation or the alternative relation primarily expresses the designer's intent, and the causal reason and the teleological reason are supplemental attributes of the chains. Therefore, even if the causal reason and the teleological reason are omitted, the design intent can be understood to some extent from only the constraint relation or the alternative relation. Furthermore, when the designer inputs the causal reason for the constraint relation or the teleological reason for the alternative relation, only simple vocabulary without structure need be prepared beforehand in order to support the capture of the design intent more clearly. Thus, because the reason itself can be expressed by simple vocabulary without structure, it is possible to choose appropriate words as the reason, by the simple estimation mechanism based on the ontology dictionary.

As stated above, in the Activity Chain Model, it is possible to support the capture of the design process information, including design intent, according to the level of details of the design process information. Consequently, it is possible to support the designer's input of the design process information at each level of the details, and to mitigate the input overhead.

2) Integration of capture, accumulation, and transfer of the design process information

In the accumulation of the design process information according to the Activity Chain Model, it becomes easier to capture the design process information because the design activities performed by the designer in the design process need only be expressed as the activity units along the time axis, and the constraint relation and the alternative relation are added between these activity units. Also it becomes 
easier to transfer the design process information because a designer can receive another designer's past design activity as activity units along the time axis, along with the constraint relations and the alternative relations between these activity units, which enable the designer to understand the design history and the design intent.

Thus, since the Activity Chain Model is suitable for both the capture and transfer of the design process information, it is possible to use the same integrated model in order to capture, accumulate, and transfer the design process information simultaneously with the propagation mechanism from the capture system to the visualization system through the database. This integrated model also enables the designer to combine the functions of capturing, accumulating, and transferring the design process information.

\section{ARCHITECTURE OF THE POET KNOWLEDGE MEDIUM}

POET (Process-Oriented Engineering Technology) Knowledge Medium implements the knowledge medium based on the ideas mentioned above as a system, and it consists of the following five subsystems (Fig. 4).

(1) Design process information database management system: EPM(engineering process manager)

(2) Design process information capturing support system: EPA(engineering process assistant)
(3) Design process information visualization system: PIVS(process information visualization system)

(4) Simulation support system: BIRD(behavior information reusable and distributed environment)

(5) Coordination control system: COOR (coordinator)

In the POET Knowledge Medium, the information unit that denotes a product unit is called "PDU (product unit)." The information unit that denotes an activity unit with the relations between the activity unit and other activity/product units is called "PCU (process unit)." The PCU consists of ontology words and links to other PCUs or PDUs, which express actions, objects, constraints, alternatives, and reasons. The POET Knowledge Medium implements the Activity Chain Model based on these information units. In the POET Knowledge Medium, the unit of change management of the PDU is the "stage" which is the time section of the design process based on the declaration by the designer. The designer's trial-and-error histories are described as branches of the stages in parallel with the time axis. The screen of the POET Knowledge Medium is shown in Fig. 5.

\section{DESIGN SIMULATION}

In this research, the authors chose the mechanical design field as the target domain of the design simulation using the POET Knowledge Medium, and the design of a DAT (digital audio tape) deck as an example. As one scenario of DAT design, the authors consider the following procedures

\section{POET Knowledge Medium}

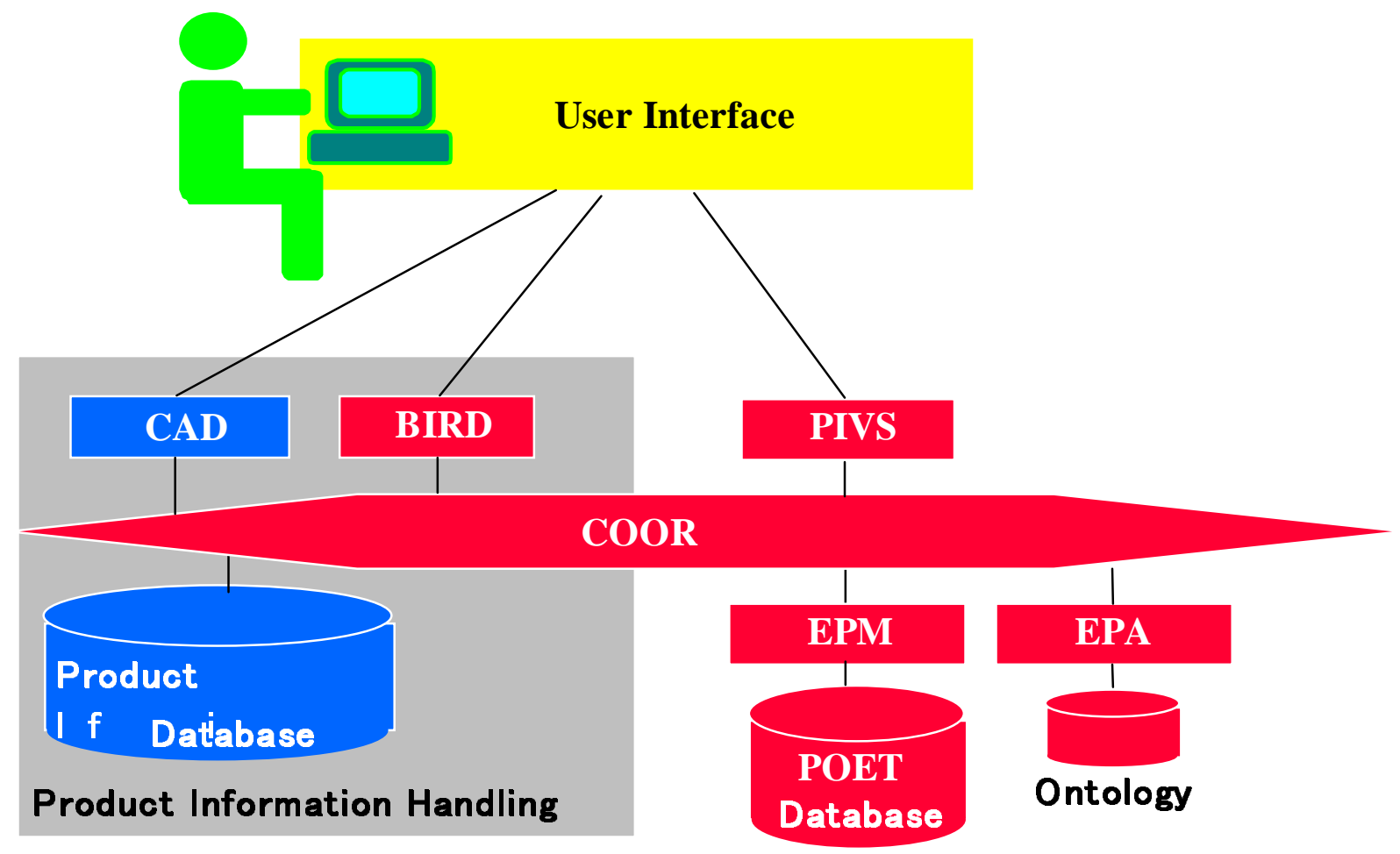

Fig. 4. POET Knowledge Medium system architecture. 


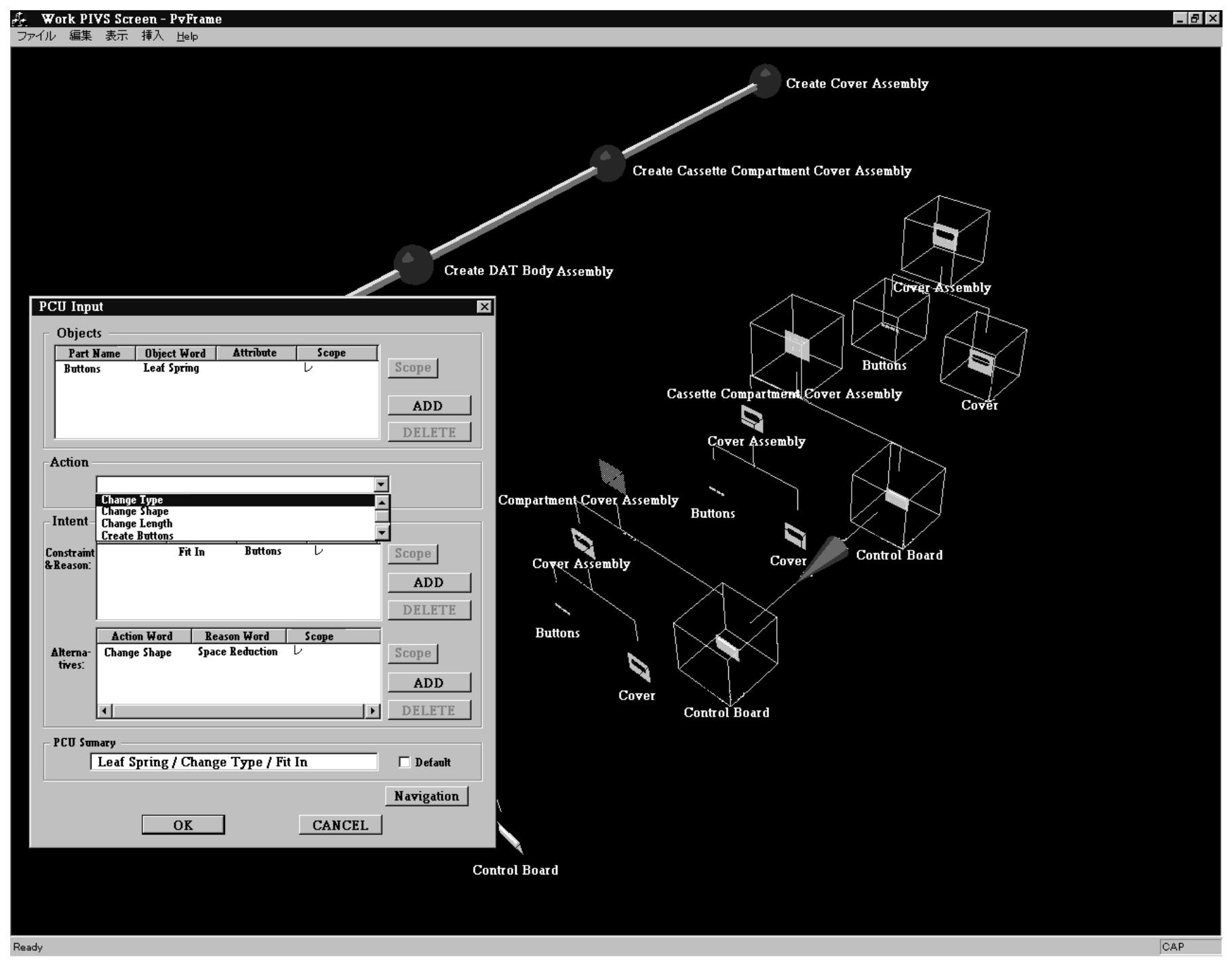

Fig. 5. Screen image of the POET Knowledge Medium.

corresponding to part of the actual DAT design process.

(1) A control board, a cassette compartment cover, and a button are created as parts.

(2) A cover assembly is created, using the cassette compartment cover and the button as children.

(3) A cassette cover assembly is created, using the control board and the cover assembly as children.

(4) An upper cabinet assembly, a lower cabinet assembly, and a mechanical deck assembly are created by reusing data for an existing model.

(5) A main body assembly is created using the upper cabinet assembly, the lower cabinet assembly, and the mechanical deck assembly as children.

(6) The BIRD carries out the simulation on the mechanical deck assembly.

(7) The shape of the guide channel of the loading link is changed based on the result of the simulation.
(8) The holes for buttons are made on the cassette compartment cover.

(9) The shape of the leaf spring of the button is changed by trial and error.

Consequently, 3 trial-and-error branches and 8 declared stages were created, and 7 representative PCUs were registered concerning the design changes. About 200 PDUs were generated in the design history information.

The authors confirmed to what extent the designer could understand the design history and the design intent based on the design process information, by simulating a case where another designer continues the work in response to a request to change the position of the button. The evaluation of the design simulation result shows that the information on the design history and the design intent can be expressed generally. The evaluation also shows that the design history and the design intent can be understood in a short time by reexperiencing the design process information. 


\section{CONCLUSIONS}

In this research, the authors proposed global design that is a new design style based on collaboration between designers beyond the physical restrictions of time and space. The POET Knowledge Medium was developed as the knowledge medium for the global design. By using knowledge media such as the POET Knowledge Medium, it is expected that a flexible design team for overseas projects can be organized dynamically, and that a global design organization can be realized. Moreover, it also is expected that knowledge and know-how concerning design can be shared on an organizational level, and that creative and effective design using ideas from past excellent designs and high-quality architecture-driven design referring to similar past designs will be realized. Furthermore, the POET Knowledge Medium in this research is considered useful for solving the designer's current problem of communication and adjustment with other designers, and for organizing an efficient design team which is flexible against changes through quick adaptation of the design to various business environments and through the utilization of the distributed design resources.

As a future research subject, it is considered important to develop the POET Knowledge Medium into more practical knowledge medium by improving the user interface and the information description model in the database, through its use in actual design work involving many designers.

\section{ACKNOWLEDGMENT}

The POET Knowledge Medium in this research was developed in the "Walk-through Engineering Knowledge Media Development" project with support from the IPA's "Advanced Software Enrichment Program" in the 1995 fiscal year.

\section{REFERENCES}

[1] K. Al-Timimi and J. MacKrell, STEP: Towards Open Systems. Ann Arbor, MI: CIMdata, 1996.

[2] J. Conklin and K C. B. Yakemovic, "A process-oriented approach to design rationale," Human-Computer Interaction, Vol. 6, pp. 357-391, 1991.
[3] J. Conklin and M. L. Begeman, "gIBIS: A hypertext tool for exploratory policy discussion," Proc. Conf. Computer-Supported Cooperative Work '88, ACM, pp. 140-152, 1988.

[4] J. Conklin, "Design rationale and maintainability," Proc. 22nd An Hawaii Int. Conf. on System Science, IEEE Computer Society Press, Vol. 2, pp. 533-539, 1989.

[5] G. Fischer, A. C. Lemke, R. McCall and A. I. Morch, "Making argumentation serve design," Human-Computer Interaction, Vol. 6, pp.393-419, 1991.

[6] G. Fischer, R. McCall and A. Morch, "Design environments for constructive and argumentative design," Proc. CHI '89, ACM, pp. 269$275,1989$.

[7] M. Fowler, UML distilled: Applying the standard object modeling language, Reading, MA: Addison-Wesley, 1997.

[8] A. C. B. Garcia and H. C. Howard, "Acquiring design knowledge through design decision justification," AI EDAM, Vol. 6, No. 1, pp. 59$71,1992$.

[9] D. R. Kuokka, J. McGuire, J. C. Weber, J. M. Tenenbaum, T. R. Gruber and G. R. Olsen, "SHADE: Knowledge-based technology for the reengineering problem," An. Rep.

[10] F. Lakin, J. Wambaugh, L. Leifer, D. Cannon and C. Sivard, "The electronic design notebook: Performing medium and processing medium," Visual Computer: Int. J. of Computer Graphics, Vol. 5, pp.214-226, 1989.

[11] S. Levy, E. Subrahmanian, S. L. Konda, R. F. Coyne, A. W. Westerberg and Y. Reich, "An overview of the n-dim environment," Technical Report EDRC-05-65-93, Engineering Design Research Center, Carnegie Mellon University, 1993.

[12] R. McCall, "PHIBIS: Procedurally hierarchical issue-based information systems," in Proc. Conf. Architecture at the International Congress on Planning and Design Theory, ASME, 1987.

[13] J. G. McGuire, D. R. Kuokka, J. C. Weber, J. M. Tenenbaum, T. R. Gruber and G. R. Olsen, "SHADE: Technology for knowledge-based collaborative engineering," J. Concurrent Eng.: Res. Appl. (CERA), Vol.1, pp. 137-146, 1993.

[14] E. Miller, "PDM today," Computer Aided Eng., Vol. 14, No. 2, pp. 3240, 1995.

[15] F. Pena-Mora, D. Sriram and R. Longcher, "Design rationale for computer-supported conflict mitigation," J. Computing in Civil Eng., Vol. 9, No. 1, pp. 57-72, 1995.

[16] T. Taura and A. Kubota, "A study on engineering history base," Res. in Eng. Design, in press.

[17] G. Toye, M. R. Cutkosky, L. J. Leifer, J. M. Tenenbaum and J. Glicksman, "SHARE: A methodology and environment for collaborative product development," Int. J. Intelligent and Cooperative Inform. Syst., Vol. 3, No. 2, pp. 129-153, 1994.

[18] G. H. von Wright, Explanation and Understanding, Cornell University Press, 1971. 